\title{
Color neutrality effects in the phase diagram of the PNJL model
}

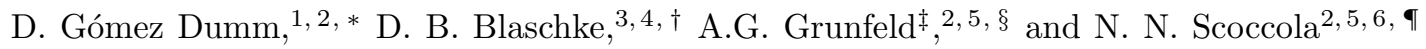 \\ ${ }^{1}$ IFLP, CONICET - Dpto. de Física, Universidad Nacional de La Plata, C.C. 67, 1900 La Plata, Argentina \\ ${ }^{2}$ CONICET, Rivadavia 1917, 1033 Buenos Aires, Argentina \\ ${ }^{3}$ Institute for Theoretical Physics, University of Wroctaw, Max Born place 9, 50204 Wroctaw, Poland \\ ${ }^{4}$ Bogoliubov Laboratory of Theoretical Physics, JINR Dubna, Joliot-Curie Street 6, 141980 Dubna, Russia \\ ${ }^{5}$ Physics Department, Comisión Nacional de Energía Atómica, \\ Av. Libertador 8250, 1429 Buenos Aires, Argentina \\ ${ }^{6}$ Universidad Favaloro, Solís 453, 1078 Buenos Aires, Argentina
}

(Dated: April 23, 2022)

\begin{abstract}
The phase diagram of a two-flavor Polyakov loop Nambu-Jona-Lasinio model is analyzed imposing the constraint of color charge neutrality. The main effect of this constraint is a coexistence of the chiral symmetry breaking ( $\chi \mathrm{SB})$ and two-flavor superconducting phases. Additional effects are a shrinking of the $\chi \mathrm{SB}$ domain in the $T-\mu$ plane and a shift of the end point to lower temperatures, but their quantitative importance is shadowed by the intrinsic uncertainties of the model. The effects can be understood in view of the presence of a nonvanishing color chemical potential $\mu_{8}$, which is introduced to compensate the color charge density $\rho_{8}$ induced by a background color gauge mean field $\phi_{3}$. At low temperatures and large chemical potentials the model exhibits a quarkyonic phase, which gets additional support from the diquark condensation.
\end{abstract}

PACS numbers: 12.29.Fe, 21.65.Qr, 25.75.Nq

\section{INTRODUCTION}

Constraints on the phase diagram of QCD under extreme conditions of high excitation (temperature) and compression (density) are of vital interest for large-scale experimental programmes with ultrarelativistic heavy-ion beams, searching for signatures of the QCD phase transitions: chiral symmetry restoration and deconfinement.

Under conditions of finite temperature $T$ and small chemical potentials $\mu$, which are probed in heavy ion collisions at SPS, RHIC and in the near future at LHC, Lattice QCD simulations have provided insight into the phase structure since the temperature-dependent change of the order parameters of the above phases, namely the chiral condensate $\langle\bar{q} q\rangle$ and the traced Polyakov loop $\Phi$, have been determined [1, 2, 3, 4]. The peak positions of the susceptibilities derived from these calculations for both transitions are remarkably coincident at the same pseudocritical temperature $T_{c}$. This might be understood in terms of general effective theory [5]. It is also remarkable that these results can be nicely described with an effective model of the NJL type, extended with the inclusion of a coupling to the Polyakov loop [6, 7, 8, 9] with a potential $\mathcal{U}(\Phi, T)$ that can be extracted from Lattice QCD simulations of the pressure in the pure gauge theory [10, 11].

The challenge for experiments as well as for theory is to extend this knowledge into the domain of finite baryon densities, where precursors of color superconductivity (pseudogap phase [12]) or even color superconducting quark matter phases themselves can occur [13, 14] and new constraints from observations of compact star properties may apply [15, 16].

Most of the effective model analyses addressing the chiral phase transition in the QCD phase diagram have been performed with NJL type models which, however, lack quark confinement and lead to an onset of finite quark densities already at unphysically low temperatures of about $50 \mathrm{MeV}$ [17], far below the deconfinement temperature of about $200 \mathrm{MeV}$ [3, 4].

The straightforward step to implement the effects of confining forces and to suppress unphysical quark degrees of freedom is to study the phase diagram of quark matter in the Polyakov loop NJL (PNJL) model. This has been carried out, e.g., in Refs. [11, 18, 19, 20, 21, 22]. In these works, however, the important question of color

\footnotetext{
$\ddagger$ Present address: Institute for Nuclear Physics, TU Darmstadt, Schloßgartenstr. 9, D-64289 Darmstadt, Germany
}

*Electronic address: dumm@fisica.unlp.edu.ar

${ }^{\dagger}$ Electronic address: blaschke@ift.uni.wroc.pl

$\S$ Electronic address: ag.grunfeld@gmail.com

IElectronic address: scoccola@tandar.cnea.gov.ar 
neutrality of quark matter has not been considered. The inclusion of neutrality constraints in the PNJL model has been taken up recently in Ref. 23]. In that paper, however, only the high-density region for a quark chemical potential $\mu=500 \mathrm{MeV}$ has been considered, and light quark masses have been neglected. It has been found that the Polyakov-loop formulation together with neutrality constraints can give rise to an increase of the critical temperature for the color superconductivity transition, in deviation from the well-known BCS relationship. An investigation of the interrelation between chiral symmetry breaking $(\chi \mathrm{SB})$ and color superconductivity, as well as a study of the effect of color neutrality without enforcing electric neutrality, is still missing. The aim of the present paper is to address these subjects, analyzing the QCD phase diagram in the framework of a generalization of the PNJL model that fulfills the constraint of color neutrality.

The article is organized as follows. In Section II we introduce the formalism for enforcing color neutrality in the PNJL model by color chemical potentials. In Section III we discuss the numerical results for the phase diagram, first in the non-superconducting case, then including color superconductivity. In the final section we present our conclusions and discuss possible consequences for the phenomenology of next heavy-ion collision experiments.

\section{FORMALISM}

The Euclidean action for the two-flavor PNJL at temperature $T$ is given by

$$
\begin{aligned}
S_{E}= & \int_{0}^{\beta} d \tau \int d^{3} x\left\{\bar{\psi}\left(-i \gamma_{\mu} D_{\mu}+\hat{m}\right) \psi-\frac{G}{2}\left[(\bar{\psi} \psi)^{2}+\left(\bar{\psi} i \gamma_{5} \vec{\tau} \psi\right)^{2}\right]\right. \\
& \left.-\frac{H}{2}\left(\bar{\psi}_{C} i \gamma_{5} \tau_{2} \lambda_{a} \psi\right)\left(\bar{\psi}_{C} i \gamma_{5} \tau_{2} \lambda_{a} \psi\right)^{\dagger}+\mathcal{U}(\Phi, T)\right\}
\end{aligned}
$$

where $\psi$ is the $N_{f}=2$ fermion doublet $\psi \equiv(u d)^{T}, \hat{m}=\operatorname{diag}\left(m_{u}, m_{d}\right)$ stands for the current quark mass matrix, and $\beta=1 / T$. For simplicity we consider the isospin symmetry limit, in which $m_{u}=m_{d}=\bar{m}_{0}$. Charge conjugated fields in Eq. (11) are defined by $\psi_{C}=\gamma_{2} \gamma_{4} \bar{\psi}^{T}$, while $\vec{\tau}$ and $\lambda_{a}$, with $a=2,5,7$, stand for Pauli and Gell-Mann matrices acting on flavor and color spaces, respectively. This Euclidean action leads to local chiral invariant current-current interactions in the quark-antiquark and quark-quark channels. The latter is expected to be responsible for the presence of a color superconducting phase in the region of low temperatures and moderate chemical potentials.

The coupling of fermions to the Polyakov loop has been implemented in Eq. (11) through the covariant derivative in the fermion kinetic term $\gamma_{\mu} D_{\mu}$, where $D_{\mu} \equiv \partial_{\mu}-i A_{\mu}$. Here the Euclidean operator $\gamma_{\mu} \partial_{\mu}$ is defined as $\gamma_{4} \frac{\partial}{\partial \tau}+\vec{\gamma} \cdot \vec{\nabla}$, with $\gamma_{4}=i \gamma_{0}$. As usual, we assume that the quarks move in a background gauge field $A_{0}=g \delta_{\mu 0} G_{a}^{\mu} \lambda^{a} / 2$, where $G_{a}^{\mu}$ are the $\mathrm{SU}(3)$ color gauge fields. Then, at the mean field level, the traced Polyakov loop is given by $\Phi=\frac{1}{3} \operatorname{Tr} \exp (i \beta \phi)$, with $\phi=i \bar{A}_{0}=$ constant. The traced Polyakov loop can be taken as an order parameter for the confinement transition. In the pure glue theory it can be associated with the spontaneous breaking of the global $Z_{3}$ center symmetry of color $S U(3), \Phi=0$ corresponding to the symmetric, confined phase [24]. Here we will work in the so-called Polyakov gauge, in which the matrix $\phi$ is given a diagonal representation $\phi=\phi_{3} \lambda_{3}+\phi_{8} \lambda_{8}$, which leaves only two independent variables, $\phi_{3}$ and $\phi_{8}$. Finally, the action (11) also includes an effective potential $\mathcal{U}$ that accounts for gauge field self-interactions.

In order to obtain the grand canonical thermodynamical potential of the PNJL model at temperature $T$ and chemical potential $\mu=\mu_{B} / 3$ we start from Eq.(10), performing a standard bosonization of the theory. This can be done by introducing fields $(\sigma, \vec{\pi})$ and $\Delta_{a}$ corresponding to the quark-antiquark and diquark channels respectively. Then we consider the mean field approximation, keeping the nonzero vacuum expectation values $\bar{\sigma}$ and $\phi$ and dropping the respective fluctuations. Concerning the superconducting vacuum, we adopt here the usual ansatz in which one has $\bar{\Delta}_{5}=\bar{\Delta}_{7}=0, \bar{\Delta}_{2}=\bar{\Delta}$. In this way, using the standard Matsubara formalism one obtains

$$
\Omega_{\mathrm{MFA}}=-\frac{T}{2} \sum_{n=-\infty}^{\infty} \int \frac{d^{3} p}{(2 \pi)^{3}} \ln \operatorname{det}\left[\beta S^{-1}(T, \mu)\right]+\frac{\bar{\sigma}^{2}}{2 G}+\frac{\bar{\Delta}^{2}}{2 H}+\mathcal{U}(\Phi, T)
$$

where the inverse propagator $S^{-1}$ is a $48 \times 48$ matrix in Dirac, flavor, color and Nambu-Gorkov spaces. The dependence of $S^{-1}$ with $T$ and $\mu\left(\mu=\mu_{B} / 3\right)$ is obtained from the $T=\mu=0$ four-momentum integrals in the fermion determinant by replacing $p_{4} \rightarrow \omega_{n}-i \tilde{\mu}$, where $\omega_{n}=(2 n+1) \pi T$ are the usual fermionic Matsubara frequencies, and $\tilde{\mu}$ stands for a complex "chemical potential" matrix in color space,

$$
\tilde{\mu}=\operatorname{diag}\left(\mu_{r}, \mu_{g}, \mu_{b}\right)+i\left(\phi_{3} \lambda_{3}+\phi_{8} \lambda_{8}\right),
$$

with

$$
\operatorname{diag}\left(\mu_{r}, \mu_{g}, \mu_{b}\right)=\mu \mathbb{1}+\mu_{3} \lambda_{3}+\mu_{8} \lambda_{8} .
$$


In general, the inclusion of color chemical potentials $\mu_{3}$ and $\mu_{8}$ is necessary to ensure color neutrality. Concerning the mean field effective potential $\mathcal{U}(\Phi, T)$, which accounts for the Polyakov loop dynamics, we use here a form that appears to be consistent with group theory constraints as well as lattice results (from which one can estimate the temperature dependence). Following Ref. [11] we take the logarithmic form of the Polyakov-loop potential, motivated by the $\mathrm{SU}(3)$ Haar measure

$$
\frac{\mathcal{U}(\Phi, T)}{T^{4}}=-\frac{1}{2} a(T) \Phi^{*} \Phi+b(T) \ln \left[1-6 \Phi^{*} \Phi+4\left(\Phi^{3}+\Phi^{* 3}\right)-3\left(\Phi^{*} \Phi\right)^{2}\right],
$$

with the corresponding definitions of $a(T)$ and $b(T)$ from [11].

It is worth to notice that in presence of the Polyakov loop the thermodynamical potential could be in general a complex quantity. As discussed in Ref. [19], in order to properly define the mean field approximation one should require that the mean field configuration provides the maximal contribution to the partition function. Then, the mean field values (order parameters) $\bar{\sigma}, \bar{\Delta}, \phi_{3}$ and $\phi_{8}$ should fulfill the coupled set of "gap equations" [19]

$$
\frac{\partial \operatorname{Re}\left[\Omega_{\mathrm{MFA}}\right]}{\left(\partial \bar{\sigma}, \partial \bar{\Delta}, \partial \phi_{3}, \partial \phi_{8}\right)}=0 \text {. }
$$

If one also imposes color charge neutrality conditions, one has two additional equations that fix the color chemical potentials $\mu_{3}$ and $\mu_{8}$. The vanishing of color charges implies

$$
\frac{\partial \operatorname{Re}\left[\Omega_{\mathrm{MFA}}\right]}{\left(\partial \mu_{3}, \partial \mu_{8}\right)}=0
$$

Finally, in order to obtain a physically meaningful solution, it has to be verified that the resulting field configuration leads to a real-valued thermodynamical potential.

At vanishing chemical potential, owing to the charge conjugation properties of the QCD Lagrangian, the mean field traced Polyakov loop $\Phi$ is expected to be a real quantity. Since $\phi_{3}$ and $\phi_{8}$ have to be real-valued [19], this condition implies $\phi_{8}=0$, and it is easy to see that the thermodynamical potential turns out to be real, as desired. In general, this need not to be the case at finite $\mu[25,26,27]$. As in Refs. 111, 19, 23] we will assume that the potential $\mathcal{U}$ is such that the condition $\phi_{8}=0$ is well satisfied for the range of values of $\mu$ and $T$ investigated here. The traced Polyakov loop is then given by $\Phi=\Phi^{*}=\left[1+2 \cos \left(\beta \phi_{3}\right)\right] / 3$. In addition, it can be seen that if $\phi_{8}=0$ then Eqs. (6) and (77) lead to $\mu_{3}=0$, leaving $\mu_{8}$ as the only potentially nonvanishing color chemical potential. This also implies that the condition $\rho_{3}=0$ is trivially satisfied. Notice that the fact that $\mu_{3}=0$, together with the ansatz chosen for $\bar{\Delta}_{a}$, allow for a residual $r-g$ color symmetry, leading to $\mu_{r}=\mu_{g}$.

The Matsubara sums can be now explicitly evaluated. One obtains

$$
\Omega_{\mathrm{MFA}}(T)=-2 \int \frac{d^{3} p}{(2 \pi)^{3}} \sum_{j=1}^{6}\left[2 T \ln \left(1+e^{-\beta E_{j}}\right)+E_{j}\right]+\frac{\bar{\sigma}^{2}}{2 G}+\frac{\bar{\Delta}^{2}}{2 H}+\mathcal{U}(\Phi, T),
$$

where the quasiparticle energies $E_{j}$ are given by

$$
\begin{aligned}
& E_{1,2}=\varepsilon_{p} \mp \mu_{b} \\
& E_{3,4}=\sqrt{\left(\varepsilon_{p} \mp \mu_{r}\right)^{2}+\bar{\Delta}^{2}}-i \phi_{3} \\
& E_{5,6}=\sqrt{\left(\varepsilon_{p} \mp \mu_{g}\right)^{2}+\bar{\Delta}^{2}}+i \phi_{3} .
\end{aligned}
$$

Here we have defined $\varepsilon_{p}=\sqrt{|\vec{p}|^{2}+M^{2}}$, being $M=\bar{m}_{0}+\bar{\sigma}$ the quark constituent mass.

The momentum integral is of course divergent. Following the usual NJL regularization prescription, we cut the integrals of the zero-point energies $E_{j}$ at a given cutoff $\Lambda$, while the logarithmic terms are integrated up to infinity. In these last integrals, however, we assume that NJL interactions vanish for energies above the cutoff, therefore we set $\bar{\sigma}=\bar{\Delta}=0$ for $|\vec{p}|>\Lambda$.

\section{RESULTS}

\section{A. Non-superconducting case}

As in most of the effective models of QCD interactions, the presence of a 2SC phase shows up at low temperatures and moderate chemical potentials. In order to study the effects of imposing the condition of color neutrality, let us 
start by considering the NJL model without quark-quark interactions $(H=0)$, which means to set $\bar{\Delta}=0$ in the effective mean field thermodynamical potential in Eq. (8). Notice that, even in the absence of color superconductivity, one still may require a nonvanishing color chemical potential $\mu_{8}$ owing to the presence of the quark interactions with the Polyakov loop. According to the discussion in the previous section, we take $\mu_{3}=0, \phi_{8}=0$, leading to a realvalued mean field thermodynamical potential. Thus the set of six relations (6) (7) is reduced to only three nontrivial equations; two for the order parameters

$$
\frac{\partial \Omega_{\mathrm{MFA}}}{\left(\partial \bar{\sigma}, \partial \phi_{3}\right)}=0
$$

and one for the constraint of color charge neutrality

$$
\frac{\partial \Omega_{\mathrm{MFA}}}{\partial \mu_{8}}=\rho_{8}=0 .
$$

If we do not include quark-quark interactions, the model includes only three free parameters, namely the quark current mass $\bar{m}_{0}$, the coupling constant $G$ and the cutoff $\Lambda$. For definiteness, to perform the numerical analysis we will use a standard set of values for these parameters, taken from Refs. 10, 11]:

$$
\bar{m}_{0}=5.5 \mathrm{MeV}, \quad G=10.1 \mathrm{GeV}^{-2}, \quad \Lambda=650 \mathrm{MeV} .
$$

We start by considering the case of $T=0$. In this limit the Polyakov loop decouples, since the mean field value $\phi_{3}$ can be removed from the momentum integrals by a shift of the integration variable $p_{4}$. One gets then two coupled equations, namely

$$
\begin{aligned}
\frac{M-\bar{m}_{0}}{G} & =\frac{M}{\pi^{2}} \int_{0}^{\Lambda} d p \frac{p^{2}}{E}\left[2 \operatorname{Sg}\left(E+\mu_{r}\right)+2 \operatorname{Sg}\left(E-\mu_{r}\right)+\operatorname{Sg}\left(E+\mu_{b}\right)+\operatorname{Sg}\left(E-\mu_{b}\right)\right], \\
0 & =\frac{2}{\sqrt{3} \pi^{2}} \int_{0}^{\Lambda} d p p^{2}\left[\operatorname{Sg}\left(E+\mu_{r}\right)-\operatorname{Sg}\left(E-\mu_{r}\right)-\operatorname{Sg}\left(E+\mu_{b}\right)+\operatorname{Sg}\left(E-\mu_{b}\right)\right] .
\end{aligned}
$$

For low values of the chemical potential $\mu$, it is easy to see that both equations are trivially satisfied for a wide range of values of $\mu_{8}$, while from Eq. (15) the value of $M$ is fixed at $M=M_{0}=324.1 \mathrm{MeV}$. Thus, as expected, the system is in a phase in which the chiral symmetry is strongly broken. These results are valid as long as $\mu \leq M_{0}$. The allowed values of $\mu_{8}$ are those which satisfy $\left|\mu_{r}\right| \leq M_{0}$ and $\left|\mu_{b}\right| \leq M_{0}$ :

$$
-\frac{M_{0}-\mu}{2} \leq \frac{\mu_{8}}{\sqrt{3}} \leq\left\{\begin{array}{lll}
\left(M_{0}+\mu\right) / 2 & \text { if } & \mu \leq M_{0} / 3 \\
M_{0}-\mu & \text { if } & M_{0} / 3<\mu<M_{0}
\end{array}\right.
$$

Now if $\mu$ is increased above $M_{0}$ the value of the effective mass $M$ slightly decreases, while from Eq. (16) one has $\mu_{8}=0$. This holds up to a critical value $\mu_{c}(T=0)=342 \mathrm{MeV}$. At this point one finds a first order transition into a normal quark matter (NQM) phase, in which the chiral symmetry is approximately restored. This behavior is shown by the $T=0$ curve in the upper panel of Fig. 1 , where we plot the value of $\bar{\sigma}=M-\bar{m}_{0}$ as function of the chemical potential $\mu$.

In order to determine the effect of the Polyakov loop one has to turn on the temperature, solving Eqs. (12,13). Let us first consider the region of low temperatures $(T \ll \mu)$ and values of $\mu$ up to $\sim M_{0}$. In this region, while the value of the effective mass is kept at $M \simeq M_{0}$, it is possible to show that the value of $\phi_{3}$ is basically determined by the minimization of the effective potential $\mathcal{U}(\Phi, T)$, which leads to $\phi_{3} \simeq 2 \pi / 3$ (or, equivalently, $\Phi \simeq 0$ ). In particular, it is interesting to consider the range $0 \leq \mu \leq M_{0} / 3$, where it is possible to perform some analytic calculations to solve Eq. (13). From this analysis, the value of $\mu_{8}$ is found to be given by the simple expression

$$
\frac{\mu_{8}}{\sqrt{3}}=2 \mu-T \ln 2 .
$$

This result allows us to find the actual value of $\mu_{8}$ in the limit $T=0$ : one has $\mu_{8} / \sqrt{3}=2 \mu$, which leads to $\mu_{r}=3 \mu$, $\mu_{b}=-3 \mu$. Notice that the condition $\mu \leq M_{0} / 3$ is necessary to be in agreement with the constraints for $\mu_{8}$ that we established above in the $T=0$ case. For low temperatures and $M_{0} / 3 \leq \mu \leq M_{0}$, it is not easy to get a relation as simple as that in Eq. (18). However, it can be seen that in the $T=0$ limit Eq. (13) implies $\mu_{r}=M_{0}$, which means $\mu_{8} / \sqrt{3}=M_{0}-\mu$. These results are shown by the $T=0$ curve in the central panel of Fig. 1 , where we quote the value of $\mu_{8}$ as function of the chemical potential. 
In this way, it has been shown that for nonzero temperatures and baryon chemical potentials the interaction between the fermions and the Polyakov loop requires the presence of a nonzero color chemical potential $\mu_{8}$ in order to keep color neutrality. This still holds in the $T=0$ limit if one demands continuity in the values of $\mu_{8}$ and $\phi_{3}$. Hence the fact that in this limit the Polyakov loop decouples does not mean that the system is blind to the color symmetry breaking induced by $\phi_{3}$.

For finite values of the temperature, the critical values of the chemical potential $\mu_{c}(T)$ can be found numerically, defining a first order phase transition line in the $T-\mu$ plane. This holds up to a given "end point" $\left(\mu_{E}, T_{E}\right)$, which is found to be located at about $(333 \mathrm{MeV}, 64 \mathrm{MeV})$ for our parameter set. For $T>T_{E}$ the transition to the normal quark matter phase proceeds as a smooth crossover. The corresponding transition line can be established by looking at the peak in the chiral susceptibility, defined by

$$
\chi \equiv-\frac{1}{2} \frac{\partial^{2} \Omega}{\partial \bar{m}_{0}^{2}} .
$$

Our numerical results are shown in Figs. 1 and 2. In Fig. 2 we represent the $T-\mu$ phase diagram, denoting by solid and dashed lines the curves corresponding to first order and crossover chiral restoration transitions respectively. For comparison we also include the corresponding curves for the PNJL model without the imposition of color neutrality. It can be seen that the first order transition line extends up to about $T_{E}=100 \mathrm{MeV}$ in this latter case. Notice that at $\mu=0$ the phase transition temperature is found to be about $255 \mathrm{MeV}$, somewhat larger than the values obtained from lattice QCD 2, 3]. This is a typical feature of local NJL models, and we do not adopt in our calculations the rescaling procedure [28] of the Polyakov-loop potential which leads to a flavor-dependent lowering of the critical temperature. It has been demonstrated that in nonlocal generalizations of the PNJL model [29] the critical temperature at $\mu=0$ is in agreement with the lattice result. In Fig. 1 we show the behavior of the effective mass $\bar{\sigma}$, the color chemical potential $\mu_{8}$ and the traced Polyakov loop $\Phi$ as functions of the chemical potential, for some representative values of the temperature. The curves for $T=100 \mathrm{MeV}$ and $T=200 \mathrm{MeV}$ show clearly the crossover transition, while for $T=300 \mathrm{MeV}$ the system is always in the normal quark matter phase (see Fig. 2). For relatively low values of $\mu$, the central and lower panels of Fig. 1 show the correlation between the deconfinement transition and the restoration of color symmetry: when the temperature is increased, the average mean field value of the traced Polyakov loop rises from 0 (confinement) towards 1 (deconfinement); this comes together with a decrease of the color chemical potential $\mu_{8}$, which has been introduced in order to recover color neutrality. The effect is diluted in presence of a large baryon chemical potential. Finally, in the upper and lower panels we have also included with dashed lines the curves corresponding to the PNJL model without color neutrality (in the central panel, these correspond simply to $\left.\mu_{8}=0\right)$. The results for the models with and without color neutrality are approximately coincident in the case of $T=300 \mathrm{MeV}$.

\section{B. Color-superconducting model}

Let us now take into account the quark-quark pairing interaction in Eq. (11), with the ansatz $\bar{\Delta}_{5}=\bar{\Delta}_{7}=0, \bar{\Delta}_{2}=\bar{\Delta}_{\text {. }}$ According to the discussion in Sect. II, we have to solve the system of equations (12), together with a fourth equation $\partial \Omega_{\mathrm{MFA}} / \partial \bar{\Delta}=0$. To proceed with the corresponding numerical calculations one has to fix the value of $H$, which cannot be directly obtained from $T=\mu=0$ phenomenology. For definiteness we will consider values of the ratio $H / G$ around 0.75, which is the value obtained from a Fierz rearrangement of the local quark-quark interaction in Eq. (11).

Our main results are shown in Figs. 3 and 4 . In Fig. 3 we show the arising $T-\mu$ phase diagrams. In order to see the qualitative dependence of the results on the value of $H$, we have considered the cases $H / G=0.7,0.75$ and 0.8 . It is seen that now the phase diagrams include in general a large region of two-flavor superconducting (2SC) phases. For large values of the chemical potential and relatively low temperatures, the system is in a $2 \mathrm{SC}$ phase in which the chiral symmetry is approximately restored. Then, at $T \simeq 150 \mathrm{MeV}$ one finds a second order phase transition into a normal quark matter (NQM) phase. In addition, for intermediate values of $\mu$, the system gets into a $2 \mathrm{SC}$ phase in which the chiral symmetry is still strongly broken $(\chi \mathrm{SB})$. Here the quarks acquire large dynamical masses, which are not substantially different from those obtained in the non-superconducting model. The size of this phase region is large for $H / G \geq 0.75$, and becomes reduced if the ratio is decreased. Once again, one finds a second order phase transition into the non-2SC phase, while the chiral restoration is driven by a first order phase transition for low temperatures, and by a smooth crossover for temperatures above a given end point. The shrinking of the $\chi \mathrm{SB}-2 \mathrm{SC}$ coexisting phase appears to be the main qualitative effect of the change in $H / G$ within the considered range.

In Fig. 4 we show the behavior of the mean field diquark condensate $\bar{\Delta}$ (left panel) and the traced Polyakov loop $\Phi$ (right panel) as functions of the chemical potential for $T=0,50 \mathrm{MeV}, 100 \mathrm{MeV}$ and $150 \mathrm{MeV}$. The curves correspond to the case $H / G=0.75$. Both the first order and the crossover chiral restoration transitions can be noticed through their effects in the diquark condensate, while the size of the diquark gap is found to be of the order of a hundred 

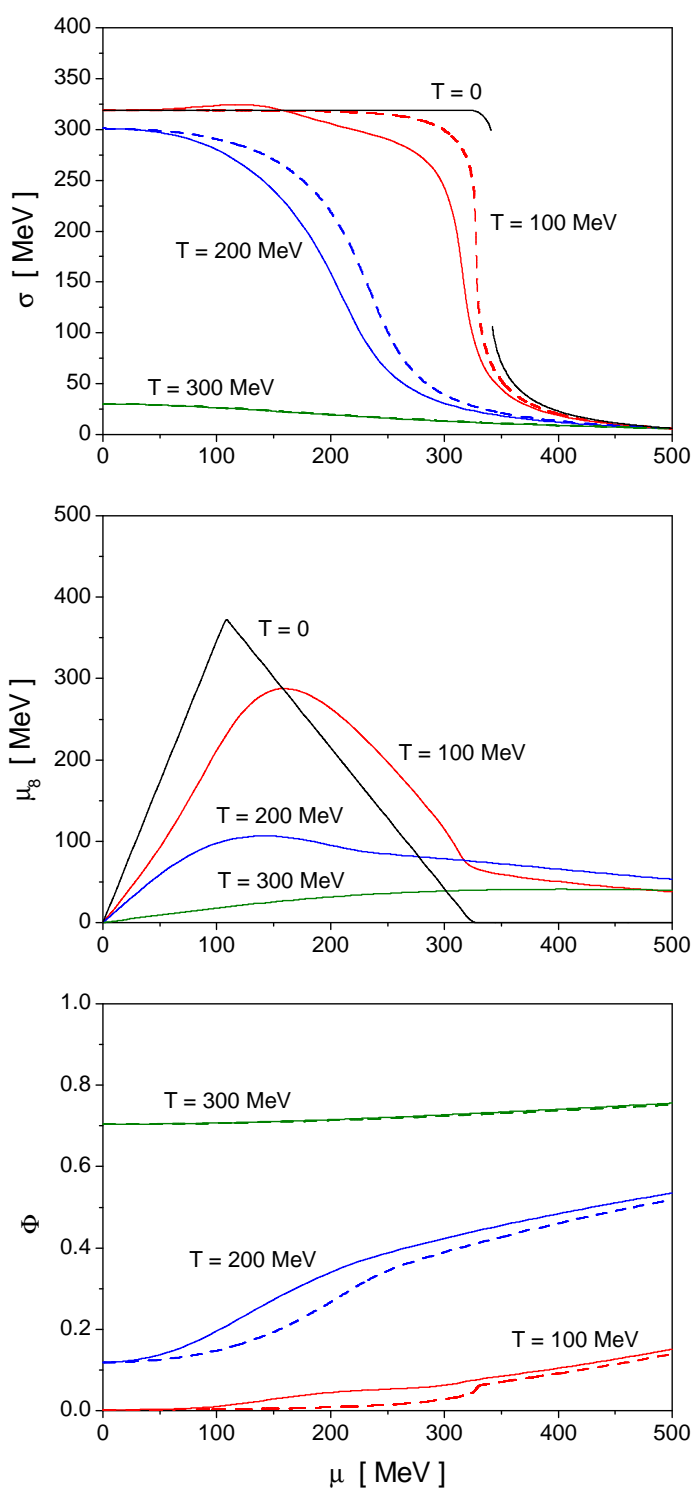

FIG. 1: Behavior of the effective mass $\bar{\sigma}$, the color chemical potential $\mu_{8}$ and the traced Polyakov loop $\Phi$ as functions of the chemical potential, for various values of the temperature, in the non-superconducting two-flavor PNJL. Solid and dashed lines correspond to the results with and without the imposition of color neutrality, respectively. Notice that in the upper and lower plots the solid and dashed curves corresponding to $T=300 \mathrm{MeV}$ are almost coincident.

$\mathrm{MeV}$ for the bulk of the temperature range (it decreases rapidly to zero near the phase border, at $T \sim 150 \mathrm{MeV}$ ). Concerning the traced Polyakov loop, it is seen that the behavior is similar to that found in the case of the nonsuperconducting model, in the sense that $\Phi \lesssim 0.15$ for temperatures below $100 \mathrm{MeV}$ and chemical potentials up to $500 \mathrm{MeV}$ (see lower panel of Fig. 1). Here the rise with $\mu$ is even less pronounced than in the non-superconducting case, since one finds a plateau in the $\chi \mathrm{SB}-2 \mathrm{SC}$ coexistence region (see curves corresponding to $T=50$ and $100 \mathrm{MeV}$ ). For comparison, the behavior of $\Phi$ in the non-superconducting model for $T=100 \mathrm{MeV}$ has also been plotted in the figure. For $T \geq 150 \mathrm{MeV}$ there is no 2SC phase, thus there is no difference between the behavior of $\Phi$ in both models. Finally, the behavior of the color chemical potential $\mu_{8}$ in both superconducting and non-superconducting models is also found to be qualitatively similar (see central panel of Fig. 11).

It has been noted earlier by Fukushima [20] that the phase diagram of the PNJL model exhibits at densities $\mu>\mu_{c}$ and low temperatures a so-called quarkyonic phase characterized by the coexistence of confinement $(\Phi \ll 1)$ and chiral symmetry restoration (see also [22]). The possibility of such a phase was suggested in Ref. [30] for large $N_{c}$ QCD where also the term was coined. In a different context it has been discussed in Ref. [31]. It is at present an 




FIG. 2: Phase diagram for the non-superconducting two-flavor PNJL model with and without the imposition of color neutrality. Solid and dashed lines correspond to first order phase transition and smooth crossover, respectively. The fat dots denote the position of the end points.
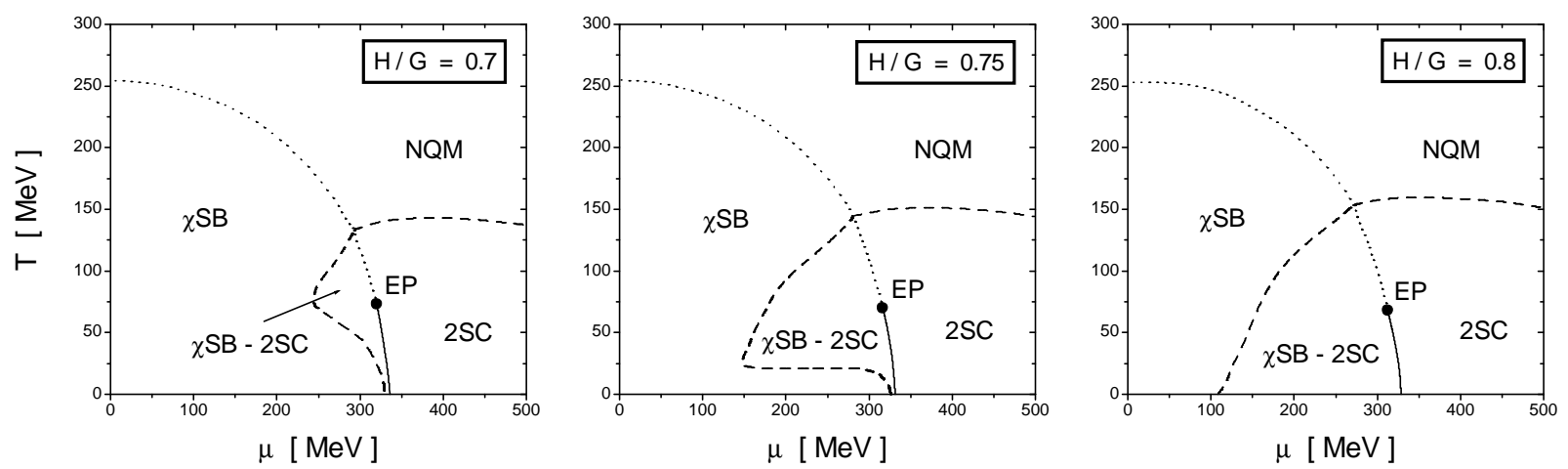

FIG. 3: $T-\mu$ phase diagrams for the neutral superconducting two-flavor PNJL model, for various values of the ratio $H / G$. Solid, dashed and dotted lines denote first order, second order and crossover phase transitions, respectively. The fat dot indicates the end point.
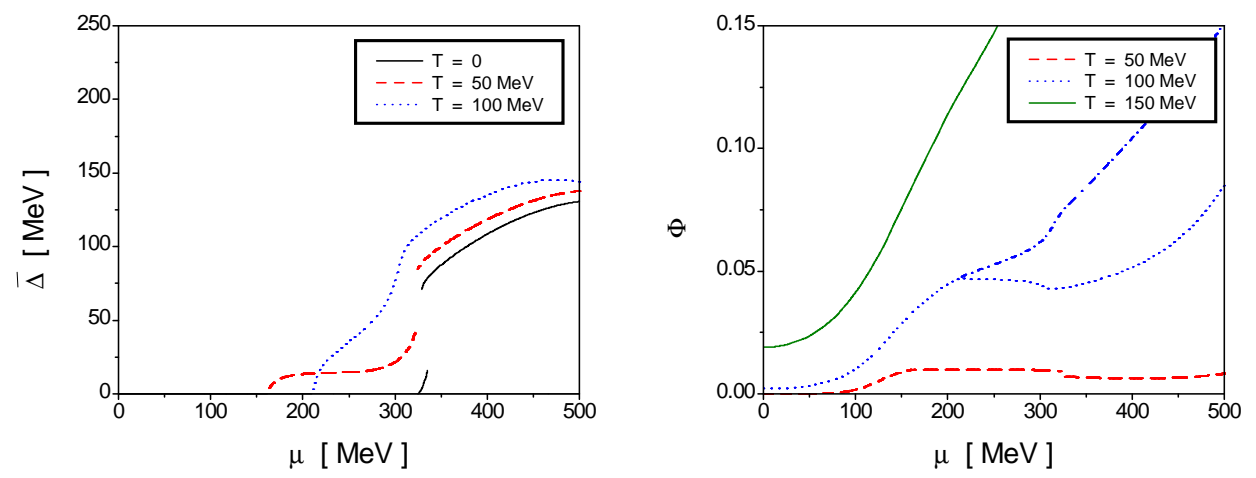

FIG. 4: Behavior of the color superconducting condensate $\bar{\Delta}$ (left) and the traced Polyakov loop $\Phi$ (right) as functions of the chemical potential, for various values of the temperature and a coupling ratio $H / G=0.75$. For comparison, the dashed-dotted line in the right panel shows the curve corresponding to $T=100 \mathrm{MeV}$ in the non-superconducting model $(H=0)$. 
open question whether a quarkyonic phase shall exist in the real world with $N_{c}=3$. As a result of the present study, we find that the requirement of color neutrality together with the occurrence of diquark condensation are consistent with the existence of a quarkyonic phase in the PNJL model. Moreover, as it is shown in the right panel of Fig. 4. the presence of diquark condensation leads to a reduction of the expectation value of the traced Polyakov loop $\Phi$ and thus enforce the confining aspects of the model.

It is important to point out that the presence of a nonvanishing Polyakov loop variable $\phi_{3}$ breaks the color $\mathrm{SU}(3)$ symmetry down to $\mathrm{SU}(2)$, so that the rotational invariance for the choice of the orientation of the $2 \mathrm{SC}$ gap under color neutrality constraints [32] might be lost. As a consequence, the above ansatz for $\bar{\Delta}_{a}$ may not correspond to the true minimum of the thermodynamical potential which instead might point to a different direction in the $\Delta_{a}$ space [33]. While accounting for such a disorientation of the 2SC condensate may lead to quantitative corrections, we believe that the qualitative structure of the phase diagram for the PNJL model under color neutrality constraints shown in the left panel of Fig. 3 remains robust. The 2SC phase might coexist with chiral symmetry breaking and confinement in the temperature range between about $50 \mathrm{MeV}$ and $100 \mathrm{MeV}$ at densities below the onset of chiral restoration with possible observable consequences in heavy-ion collision experiments, e.g., due to diquark-antidiquark annihilation into lepton pairs [34]. Note that with the coexistence of $\chi \mathrm{SB}$ and 2SC phases the condition for Bose-Einstein condensation (BEC) of diquark bound states is fulfilled and the chiral restoration transition in the above temperature window may therefore be characterized as a BEC-BCS crossover transition where the diquark bound states undergo a Mott effect to unbound, but resonant diquark Cooper pairs [35, 36]

\section{CONCLUSIONS}

In this paper we have explored the consequences of imposing the color neutrality constraint within the framework of the standard mean field treatment of the PNJL model at finite chemical potential. We have found that the fulfillment of such a constraint leads to some changes in the phase diagram of the model, as e.g. a shrinking of the $\chi \mathrm{SB}$ domain in the $T-\mu$ plane and a shift of the end point to lower temperatures. The most noticeable effect turns out to be the presence of a coexistence region of $\chi \mathrm{SB}$ and $2 \mathrm{SC}$ phases, which may have consequences for possible applications of the model in heavy-ion collision experiments.

The effects can be understood due to the nonvanishing color chemical potential $\mu_{8}$, which is introduced to compensate the color charge $\rho_{8}$ induced by the color background field $\phi_{3}$. For a given baryochemical potential $\mu, \mu_{8}>0$ reduces the chemical potential of blue quarks, while simultaneously increasing that of red and green ones. The net effect is a shift of the $\chi \mathrm{SB}$ phase border to lower $\mu$ values than without the color neutrality constraint, due to the nonlinear dependence of the quark mass gap on the chemical potential close to its critical value for a given, not too low temperature. It is seen that this effect is relatively slight, taking into account the theoretical uncertainty that can be in general expected from an effective quark model. In addition, the increase of chemical potentials of red and green quarks, which pair in the 2SC channel, entails an effective lowering of the critical value of $\mu=\mu_{c}$ for the onset of the 2SC phase, again for not too low temperatures. One finds in this way a $\chi \mathrm{SB}-2 \mathrm{SC}$ coexisting phase, whose size depends on the value of the parameter $H$ that drives the quark-quark interaction. We notice that the 2SC border has been obtained after choosing a given ansatz for the mean field values $\bar{\Delta}_{a}$. The actual orientation of the 2SC gap in color space under the presence of the Polyakov loop is a subject that deserves further study. Moreover, it is worth to point out that at finite densities there is no fundamental need to be restricted to Lorentz invariant condensates, thus more general mean field configurations could be considered.

Signatures of the coexisting $\chi \mathrm{SB}$ and $2 \mathrm{SC}$ phases might become apparent in not too energetic heavy-ion collision experiments (about 3-5 GeV/nucleon), as they are possible at the future facilities FAIR-CBM (Darmstadt), NICAMPD (Dubna) and J-PARC (Japan). Such a mixed phase can give rise, e.g., to an enhancement of low-mass dilepton production due to diquark-antidiquark annihilation. It is a realization of the BEC-BCS crossover in quark matter. While such a transition has previously been discussed in the NJL model by artificially increasing the diquark coupling to large values 36, 37, 38], which can result in conflicts with the nuclear matter ground state, in the present PNJL model the $\chi \mathrm{SB}-2 \mathrm{SC}$ coexisting phase has been obtained without any artificial tuning of parameters. It just occurs as a consequence of color neutrality in a domain of temperatures and chemical potentials around the critical end point of the first order chiral phase transitions for the standard Fierz-value of the diquark coupling, $H / G=0.75$, without affecting the nuclear matter ground state at $T=0$.

Finally, it has been shown that the coexistence of confinement and chiral symmetry restoration characterizing a quarkyonic phase in the PNJL model at finite densities and low temperatures gets even reinforced due to color superconductivity. The occurrence of diquark condensation reduces the expectation value of the traced Polyakov loop and thus acts towards strengthening the confinement aspect of the model. 


\section{Acknowledgments}

This work has been supported in part by CONICET and ANPCyT (Argentina), under grants PIP 6009, PIP 6084 and PICT04-03-25374. D.B. acknowledges support from the Polish Ministry of Science and Higher Education under contract No. N N202 0953 33. He is grateful for the hospitality of the Institute for Nuclear Theory at the University of Washington and for partial support from the Department of Energy during the completion of this work.

[1] F. Karsch and E. Laermann, Phys. Rev. D 50, 6954 (1994) arXiv:hep-lat/9406008.

[2] F. Karsch, J. Phys. Conf. Ser. 46, 122 (2006) arXiv:hep-lat/0608003.

[3] M. Cheng et al., Phys. Rev. D 74, 054507 (2006) arXiv:hep-lat/0608013.

[4] M. Cheng et al., Phys. Rev. D 77, 014511 (2008) arXiv:0710.0354 [hep-lat]].

[5] A. Mocsy, F. Sannino and K. Tuominen, Phys. Rev. Lett. 92, 182302 (2004) arXiv:hep-ph/0308135; T. Kahara and K. Tuominen, Phys. Rev. D 78, 034015 (2008) arXiv:0803.2598 [hep-ph]].

[6] K. Fukushima, Prog. Theor. Phys. Suppl. 151 (2003) 171.

[7] K. Fukushima, Phys. Lett. B 591, 277 (2004) arXiv:hep-ph/0310121.

[8] K. Fukushima, Phys. Rev. D 68, 045004 (2003) arXiv:hep-ph/0303225].

[9] K. Fukushima, Phys. Lett. B 553, 38 (2003) arXiv:hep-ph/0209311.

[10] C. Ratti, M. A. Thaler and W. Weise, Phys. Rev. D 73 (2006) 014019.

[11] S. Roessner, C. Ratti and W. Weise, Phys. Rev. D 75, 034007 (2007) arXiv:hep-ph/0609281.

[12] M. Kitazawa, T. Koide, T. Kunihiro and Y. Nemoto, Phys. Rev. D 65, 091504 (2002) arXiv:nucl-th/0111022 ; M. Kitazawa, T. Koide, T. Kunihiro and Y. Nemoto, Phys. Rev. D 70, 056003 (2004) arXiv:hep-ph/0309026.

[13] M. Buballa, Phys. Rept. 407, 205 (2005) arXiv:hep-ph/0402234.

[14] M. G. Alford, A. Schmitt, K. Rajagopal and T. Schäfer, arXiv:0709.4635 [hep-ph].

[15] T. Klähn et al., Phys. Lett. B 654, 170 (2007) arXiv:nucl-th/0609067.

[16] M. Alford, D. Blaschke, A. Drago, T. Klähn, G. Pagliara and J. Schaffner-Bielich, Nature 445, E7 (2007) arXiv:astro-ph/0606524.

[17] P. Zhuang, J. Hüfner and S. P. Klevansky, Nucl. Phys. A 576, 525 (1994).

[18] C. Sasaki, B. Friman and K. Redlich, Phys. Rev. D 75, 074013 (2007) arXiv:hep-ph/0611147.

[19] S. Roessner, T. Hell, C. Ratti and W. Weise, arXiv:0712.3152 [hep-ph].

[20] K. Fukushima, Phys. Rev. D 77, 114028 (2008) [Erratum-ibid. D 78, 039902 (2008)] arXiv:0803.3318 [hep-ph]].

[21] M. Ciminale, R. Gatto, N. D. Ippolito, G. Nardulli and M. Ruggieri, Phys. Rev. D 77, 054023 (2008) arXiv:0711.3397 [hep-ph]].

[22] H. Abuki, R. Anglani, R. Gatto, G. Nardulli and M. Ruggieri, arXiv:0805.1509 [hep-ph].

[23] H. Abuki, M. Ciminale, R. Gatto, G. Nardulli and M. Ruggieri, Phys. Rev. D 77, 074018 (2008) [arXiv:0802.2396 [hep-ph]].

[24] R. D. Pisarski, Phys. Rev. D 62, 111501 (2000) arXiv:hep-ph/0006205.

[25] H.-T. Elze, D. E. Miller and K. Redlich, Phys. Rev. D 35, 748 (1987).

[26] A. Dumitru, R. D. Pisarski and D. Zschiesche, Phys. Rev. D 72, 065008 (2005) arXiv:hep-ph/0505256.

[27] K. Fukushima and Y. Hidaka, Phys. Rev. D 75, 036002 (2007) arXiv:hep-ph/0610323.

[28] B. J. Schaefer, J. M. Pawlowski and J. Wambach, Phys. Rev. D 76, 074023 (2007) arXiv:0704.3234 [hep-ph]].

[29] D. Blaschke, M. Buballa, A. E. Radzhabov and M. K. Volkov, arXiv:0705.0384 [hep-ph]; G. A. Contrera, D. Gomez Dumm and N. N. Scoccola, Phys. Lett. B 661, 113 (2008) [arXiv:0711.0139 [hep-ph]].

[30] L. McLerran and R. D. Pisarski, Nucl. Phys. A 796, 83 (2007) arXiv:0706.2191 [hep-ph]].

[31] L. Y. Glozman, arXiv:0803.1636 [hep-ph].

[32] M. Buballa and I. A. Shovkovy, Phys. Rev. D 72, 097501 (2005) arXiv:hep-ph/0508197.

[33] D. Blaschke, D. Gomez Dumm, A. G. Grunfeld and N. N. Scoccola, arXiv:hep-ph/0507271.

[34] T. Kunihiro, M. Kitazawa and Y. Nemoto, arXiv:0711.4429 [hep-ph].

[35] G. f. Sun, L. He and P. Zhuang, Phys. Rev. D 75, 096004 (2007) arXiv:hep-ph/0703159.

[36] D. Zablocki, D. Blaschke and R. Anglani, arXiv:0805.2687 [hep-ph].

[37] M. Huang, P.-F. Zhuang and W.-Q. Chao, Phys. Rev. D 65, 076012 (2002).

[38] M. Kitazawa, D. H. Rischke and I. A. Shovkovy, Phys. Lett. B 663, 228 (2008). 